Cahiers $d u$ MONDE RUSSE

\section{Cahiers du monde russe}

Russie - Empire russe - Union soviétique et États indépendants

$48 / 1 \mid 2007$

Émigrations au début du XXe siècle

\title{
Eux et nous: la guerre froide dans les histoires drôles soviétiques
}

Andreï Kozovoï

\section{OpenEdition}

Journals

Édition électronique

URL : https://journals.openedition.org/monderusse/8989

DOI : 10.4000/monderusse.8989

ISSN : $1777-5388$

Éditeur

Éditions de l'EHESS

\section{Édition imprimée}

Date de publication : 15 janvier 2007

Pagination : 137-152

ISBN : 978-2-7132-2146-0

ISSN : $1252-6576$

Référence électronique

Andreï Kozovoï, «Eux et nous : la guerre froide dans les histoires drôles soviétiques », Cahiers du monde russe [En ligne], 48/1 | 2007, mis en ligne le 01 janvier 2007, consulté le 02 septembre 2022. URL : http://journals.openedition.org/monderusse/8989; DOI : https://doi.org/10.4000/monderusse. 8989 
chercher : repérer : avancer

Cet article est disponible en ligne à l'adresse :

http://www.cairn.info/article.php?ID REVUE=CMR\&ID NUMPUBLIE=CMR 481\&ID ARTICLE=CMR 4810137

\title{
Eux et nous: la guerre froide dans les histoires drôles soviétiques
}

\author{
par Andreï KOZOVOÏ
}

\section{| Editions de l'EHESS | Cahiers du monde russe}

\author{
$2007 / 1$ - Vol 48 \\ ISSN 1252-6576 | ISBN 9782713221460 | pages 137 à 152
}

Pour citer cet article :

—Kozovoï ., Eux et nous : la guerre froide dans les histoires drôles soviétiques, Cahiers du monde russe 2007/ 1, Vol 48 , p. $137-152$.

Distribution électronique Cairn pour les Editions de l'EHESS.

(C) Editions de l'EHESS. Tous droits réservés pour tous pays.

La reproduction ou représentation de cet article, notamment par photocopie, n'est autorisée que dans les limites des conditions générales d'utilisation du site ou, le cas échéant, des conditions générales de la licence souscrite par votre établissement. Toute autre reproduction ou représentation, en tout ou partie, sous quelque forme et de quelque manière que ce soit, est interdite sauf accord préalable et écrit de l'éditeur, en dehors des cas prévus par la législation en vigueur en France. Il est précisé que son stockage dans une base de données est également interdit. 


\title{
EUX ET NOUS : LA GUERRE FROIDE DANS LES HISTOIRES DRÔLES SOVIÉTIQUES
}

\begin{abstract}
L'historien qui voudrait restituer l'opinion publique soviétique se heurtera immanquablement à des obstacles de taille : sondages rares et peu fiables, accès particulièrement difficile aux rapports des archives du KGB sur l' «état de l'opinion », difficulté à brosser un tableau d'ensemble à partir des sources épistolaires, éparpillement des témoignages oraux. Demeure un matériau ayant trait à la culture populaire et d'accès facile : l'histoire drôle politique, en russe anekdot ${ }^{1}$. Cette « histoire imaginaire faisant appel à (ou qui sous-entend) un contexte politique et/ou des personnages bien réels » peut-elle se révéler dans ce contexte la source, certes secondaire, mais néanmoins précieuse, qui permettrait de suppléer dans une certaine mesure aux données manquantes ?
\end{abstract}

\footnotetext{
* Andreï Kozovoï a soutenu en décembre 2006 une thèse d'histoire à l'Université de Paris, sous la direction du professeur Marie-Pierre Rey : « Présences des États-Unis en URSS, 19751985 : le grand public soviétique et les pratiques périaméricaines des pouvoirs ». Ses axes de recherches portent sur l'antiaméricanisme en Russie, les relations soviéto-américaines dans les années 1970-1980 et le cinéma de guerre froide.
}

1. Nous utiliserons plus loin le terme « anecdote », même s'il ne recouvre pas en français la même réalité que le mot russe.

2. Cet article est la version élargie de notre communication (18 septembre 2004) au symposium sur «L'histoire culturelle et sociale de la Russie, XVIII'-XXe siècles » organisé par le Centre franco-russe pour les sciences sociales (Moscou) dont nous remercions l'ancien directeur, Alexis Berelowitch. Nous exprimons également notre gratitude à Aleksandra Arhipova (RGGU, Moscou) et Amandine Regamey (Paris I, SELVA) pour leurs précieux conseils. Cette dernière est notamment l'auteur d'une thèse publiée en 2007 sous le titre Prolétaires de tous pays, excusez-moi! (P. : Buchet-Chastel). Pour les anecdotes russes, on consultera d'abord l'ouvrage récent de E. Šmeleva et A. Šmelev Russkij anekdot : tekst i rečevoj žanr [L'anecdote russe : texte et niveaux de langue], M. : Jazyki slavjanskoj kul'tury, 2002. Emil A. Draitser (né en 1937), Russe émigré aux États-Unis dans les années 1970, a écrit plusieurs ouvrages sur les anecdotes : Forbidden Laughter : Soviet Underground Jokes..., Los Angeles : Almanac Pub. House, 1978, Taking Penguins to the Movies : Ethnic Humor in Russia, Detroit : Wayne State University Press, 1998 et Making War, Not Love : Gender and Sexuality in Russian Humor, New York : St Martin's Press, 1999. 
Le moins que l'on puisse dire, c'est que le corpus d'anecdotes hérité de l'époque soviétique n'a justement rien d'anecdotique : un recueil ancien (1986) en a répertorié 1001, mais il ne s'agit que d'une sélection ${ }^{3}$. Cela s'explique par le fait que la couche de la population soviétique que nous connaissons le mieux, les habitants des grandes villes et en particulier les milieux instruits, a depuis toujours baigné dans une culture de l'humour politique, ce qui n'a pas d'équivalent dans les pays occidentaux au $\mathrm{XX}^{\mathrm{e}}$ siècle ${ }^{4}$. Étudier cela pour la période de la « guerre fraîche », comprise de manière large entre 1975, année à la fois de l'apogée et de la fin de la «détente », et 1985, marquée par l'avènement de Gorbačev, peut s'avérer enrichissant à plusieurs titres. Ces années sont un nouvel âge d'or de l'anecdote, qui met à profit un contexte intérieur où se combinent crises économique, politique et idéologique, sur fond d'un certain laxisme, sans doute calculé, du pouvoir ${ }^{5}$. Cette décennie est aussi marquée dans la propagande officielle par un nouveau cycle de démonisation ${ }^{6}$ du principal et plus vieil ennemi idéologique de l'URSS, les États-Unis 7 . Il n'est donc guère étonnant que ceux-ci soient l'un des protagonistes les plus exploités des anecdotes politiques.

3. Il s'agit de l'ouvrage classique compilé par le dissident Julius Telesin : 1001 izbrannyj sovetskij političseskij anekdot,Tenafli : Ermitaž, 1986, qui nous a servi de référentiel principal pour la datation des anecdotes.

4. Šmeleva et Šmelev, Russkij anekdot..., p. 12-13.

5. Pour l'attitude du pouvoir soviétique à l'égard de l'anecdote et l'utilisation de l'anecdote comme source, voir le chapitre IV de la thèse d'Amandine Regamey : « Le pouvoir soviétique avait-il peur du rire ? » et la thèse de Rosa Magnusdottir : Keeping up Appearences : How The Soviet State Failed to Control Popular Attitudes Toward the United States of America, 19451959, Chapel Hill, 2006, p. 86-87. Si, sous Stalin, un individu pouvait perdre son emploi, voire sa vie, pour avoir raconté des anecdotes, les officiels semblent avoir été bien plus tolérants à partir de Brežnev, voir Leonid Mlecin, Brežnev, M. : Prospect, 2005. Certaines histoires découleraient directement de récits de rencontre entre les dirigeants qui ne pouvaient être connus que de l'entourage proche - ce qui pose la question du vecteur de diffusion des anecdotes : je fais en particulier référence à une histoire mettant en scène Brežnev et Nixon qui parlent de leurs secrétaires respectives, conversation qui aurait vraiment eu lieu, voir A. Majsurjan, Drugoj Brežnev, M. : Vagrius, 2004, p. 48. En ce qui concerne le bref passage au pouvoir d'Andropov (novembre 1982-février 1984) et de Černenko (février 1984-mars 1985), peu de choses sont certaines à l'heure actuelle, sinon que si la tolérance de Brežnev et sa personnalité contribuaient au succès et à la richesse du genre, l'allergie d'Andropov aux anecdotes antisoviétiques a pu freiner dans une certaine mesure leur propagation, voir le témoignage de I. E. Sinicyn : Andropov vblizi. Vospominanija o vremenah «ottepeli » $i$ «zastoja », M. : IIK Rossijskaja gazeta, 2004, p. 67. Personne ne fut arrêté pour «diffusion orale d'anecdotes » au cours de notre période, même si l'article 190 du Code pénal est toujours en vigueur: « клеветнические измышления, порочащие государственный или общественный строй » [inventions mensongères ternissant la réputation du régime soviétique], pour lesquelles les anekdotisty encouraient trois ans de réclusion. Nous remercions l'historien russe Nikita Petrov pour cette précision.

6. Nous empruntons le terme «démonisation» à Jonathan Becker, Soviet and Russian Press Coverage of the United States : Press, Politics, and Identity in Transition, 2e éd., New York : St. Martin's Press, 2002,p. 112 : « use of language, symbols and images which depict a group as aggressive, physically menacing and threatening to the values and norms of the demonizing group, which instil fear and create negative emotions, and which therefore legitimate any action, internal or external, to protect the demonizing group from the demonized ».

7. La propagande antiaméricaine, dans sa forme la plus obsessionnelle, n'a jamais cessé dans les médias soviétiques depuis la période 1948-1949, sous forme de cycles - l'antiaméricanisme 
Que savent les Soviétiques de l'Amérique? Une majorité qui n'y est jamais allée se fait son opinion à travers le prisme de l'information officielle et des préjugés de chacun. Certes, l'information officielle n'est pas, dans ces années, l'unique source de référence. Une concurrence existe, celle par exemple des radios étrangères qui, bien que brouillées, sont écoutées par bon nombre de Russes qui ne sont pas tous dissidents ${ }^{8}$. Le rapport des Soviétiques à l'information officielle est de fait au cœur de notre problématique : la popularité de l'anecdote politique à protagonistes américains reflète-t-elle le discrédit de la propagande antiaméricaine, fournit-elle par là un indice de la perception de la guerre froide par le public ?

$\mathrm{Au}$ sein d'un corpus de 94 anecdotes réunies par nos soins ${ }^{9}$, nous avons distingué trois principaux types : deux mondes que tout sépare, leur rivalité, leurs contacts au sommet. Chaque type sera décrit, replacé dans le contexte international tel qu'il apparaît dans les médias soviétiques à l'époque. Nous nous efforcerons de dater les anecdotes, tâche des plus délicates tant le matériau s'avère malléable, s'adaptant au gré du contexte. Nous nous interrogerons enfin, à partir de ces prémices, sur la diffusion des histoires et leur impact sur l'opinion publique.

« débridé » succédant à un antiaméricanisme « limité ». La période 1975-1985 est marquée par plusieurs cycles : les relations soviéto-américaines commencent à se dégrader sous la présidence de Gerald Ford (1974-1977), puis se dégradent considérablement sous Jimmy Carter (1977-1981), champion du combat pour les droits de l'homme. L'apogée des tensions est atteint sous le premier mandat de Ronald Reagan (1981-1985), dont la rhétorique ouvertement anticommuniste s'accompagne d'actions fortes sur le plan international. L'arrivée au pouvoir de Mihail Gorbačev en mars 1985, bien qu'elle ne marque pas d'emblée une volonté de faire table rase du passé antiaméricain, coïncide avec de nouvelles orientations de la politique étrangère américaine et inaugure donc un nouveau cycle.

8. Voir l'ouvrage de Tristan Mattelart, Le Cheval de Troie de l'audiovisuel : Le rideau de fer à l'épreuve des radios et télévisions transfrontalières, Grenoble : PUG, 1995.

9. Réunir l'intégralité des histoires qui ont circulé à l'époque serait une tâche titanesque, mais nous pouvons toutefois en constituer un échantillon suffisamment large pour être représentatif. Les «lieux de conservation» des anecdotes sont d'au moins trois types; les témoins de l'époque appartiennent au premier groupe. Les effets du temps sur la mémoire sont connus et, outre la confirmation orale que telle histoire a bien circulé à une période donnée, l'apport de ces témoignages n'est pas substantiel. Le second «lieu» est constitué de recueils d'anecdotes parus au cours de la «guerre fraîche », ou peu après. Parmi les plus connus, outre celui de Telesin déjà mentionné, citons le livre de S. Karačevcev, 1200 anekdotov [1200 anecdotes], 2e éd. P. : 1978 ; celui de A. Verner, Rossija smeetsja nad SSSR [La Russie se moque de l'URSS], P. : Ritm, 1980 ; et enfin, ouvrage unique, parce qu'il tente de dater les anecdotes : Dora Štuman et Sergej Tiktin, Sovetskij Sojuz v zerkale političeskogo anekdota [L'Union soviétique dans le miroir de l'anecdote politique], 2e édition, Jérusalem : Express, 1987 (1 1 $^{\text {re }}$ édition, Londres, OPI, 1985). Enfin, incontournable aujourd'hui, l'internet : deux des sites les plus connus sont www.prikol.ru et www.anekdot.ru. Nous aurons recours à des histoires qui peuvent être un tant soit peu datées et écarterons les « inclassables » dans lesquelles les Américains et les États-Unis ne sont pas pertinentes par rapport à notre propos. L'idée selon laquelle des employés du KGB auraient été chargés d'inventer des anecdotes pour contrer celles « de la CIA » est aussi répandue qu'invérifiable, les archives du FSB n'étant pas consultables. 


\section{Deux mondes distincts}

Les anecdotes de cette catégorie sont difficiles à dater avec précision : les recueils permettent d'inférer qu'elles circulent au cours de la période que nous étudions. Toutes mettent en scène les différences fondamentales entre les deux pays, tant du point de vue politique et idéologique, que culturel et social. C'est le cas de trois anecdotes «métaphoriques » qui, à l'instar de celle qui suit, opposent un Occident civilisé à une Union soviétique « à part » :

Американец, англичанин и русский хвалятся, что заставят кошку съесть горчицу. Американец хватает кошку и запихивает горчицу ей в пасть. « Это насилие! » протестует русский. Англичанин кладет горчицу между двумя кусками колбасы, и кошка съедает. «Это обман! » протестует русский, после чего мажет горчицей кошке под хвостом, и кошка с воем вылизывает. « Обратите внимание, говорит русский, добровольно и с песней ». ${ }^{10}$

Ce récit s'apparente à une parabole dont le but est de dénoncer le mensonge de la propagande soviétique. Le Russe dénonce les Anglo-Saxons à la manière des slogans utilisés par les médias soviétiques, pour finalement démontrer que le commentaire par lequel il accompagne sa stratégie n'est qu'un habillage dérisoire de la réalité. L'Américain ou l'Anglais n'ont qu'un rôle de faire-valoir, et le trait de caractère qu'on leur prête ici (faire usage de la violence pour arriver à ses fins outil de l'impérialisme parmi d'autres de la propagande antiaméricaine) n'est pas le sujet central de l'histoire. Néanmoins une différence entre l'URSS et l'Occident perce en arrière-plan.

La majorité des histoires de ce premier groupe est cependant beaucoup plus explicite :

Советская рабочая делегация в Америке.

- Это чей завод?

- Форда.

- А чьи это автомобили стоят?

- Рабочих.

Американская рабочая делегация в СССР.

- Чей это завод?

- Он принадлежит рабочим.

- А чей это автомобиль стоит?

- Директора завода. ${ }^{11}$

10. Un Américain, un Anglais et un Russe se vantent de faire avaler de la moutarde à un chat. L'Américain se saisit du chat et lui met la moutarde dans la gueule. "C'est de la contrainte ! », proteste le Russe. L'Anglais met la moutarde entre deux morceaux de saucisson et le chat les avale. «C'est de la tromperie ! », proteste le Russe, qui après cela badigeonne de moutarde le derrière du chat ; le chat se nettoie en hurlant. « Voyez vous-même, dit le Russe, il en a mangé de son plein gré, et ce en chantant ».

11. Une délégation ouvrière soviétique aux États-Unis :

- À qui appartient cette usine?

- À Ford. 
Le mensonge soviétique est encore une fois au centre de l'anecdote qui voit dans l'URSS le reflet déformé de la réalité américaine. Il s'agit cette fois-ci de dénoncer le prétendu « pouvoir du peuple » en URSS. Le monde américain, représenté par le constructeur Ford, symbole honni du capitalisme repris de la propagande stalinienne, s'avère en réalité bien plus démocratique qu'une entreprise soviétique qui affiche un humanisme de façade. Bien souvent, l'Américain est dans le rôle du candide dont l'incompréhension révèle les tares soviétiques ; les Américains apparaissent pour la plupart comme des gens simples, appartenant au monde ouvrier, ce qui renforce la crédibilité du message général.

Dans ce groupe, l'Américain s'oppose au Russe et les États-Unis sont ce que l'URSS n'est pas : libres, incapables de mentir. Le choix des États-Unis comme protagoniste s'explique : l'Amérique n'est-elle pas le pays occidental auquel on se mesure depuis les origines de l'URSS, celui qu'on ambitionne de « rattraper et dépasser », le seul digne d'être un rival, celui qui motive la plupart des décisions en matière de politique étrangère ? Ce choix ne date pas des années 1970, on s'en doute, c'est l'aboutissement d'une longue maturation que l'on peut faire remonter à la fin du XIXe siècle, mais surtout à la fin de la Seconde Guerre mondiale, quand l'URSS et les États-Unis se posaient comme deux superpuissances ${ }^{12}$. Ceci devient visible surtout après la mort de Stalin et est véhiculé dans de nombreuses anecdotes qui émergent sans doute à partir de 1956, dans le sillage d'un XX $\mathrm{X}^{\mathrm{e}}$ Congrès ressenti comme autorisant une expression plus libre. Pour les Soviétiques, l'événement détonateur de la diffusion de ce type d'anecdotes serait l'exposition américaine de Moscou en juillet 1959 au cours de laquelle Hruščev et le vice-président Nixon ont un échange assez vif sur les avantages et les inconvénients de leurs systèmes respectifs - thème idéal des histoires de ce genre. On peut également avancer une autre hypothèse. Lors du Festival de la jeunesse de l'été 1957, les Soviétiques découvrent en grand nombre dans les rues de Moscou de jeunes Américains en chair et en os. Ils auraient alors pu se rendre compte du caractère suranné des stéréotypes de la propagande. Les années 1960 sont celles de la «découverte» de l'Amérique par l'URSS « d'en bas » et se traduisent par une américanophilie qui transparaît tant dans l'immense succès de Hemingway que dans la mode des prénoms américains, mais c'est dans les années 1970, lors de la détente initiée par l'administration Nixon, que la volonté de découvrir la «vraie » Amérique prend une réelle ampleur :

- Et ces voitures ?

- Aux ouvriers.

Une délégation ouvrière américaine en URSS :

- À qui appartient cette usine?

- Aux ouvriers.

- Et cette voiture?

- Au directeur de l'usine.

12. Alan M. BALL, Imagining America : Influence and Images in Twentieth-Century Russia, Lanham, MD ; Oxford : Rowman \& Littlefield, 2003. 
Советский гражданин бросается к машине американского посла и прокалывает шину.

- Воздухом свободы подышать захотелось! - объясняет он. ${ }^{13}$

On joue ici de l'effet de surprise : au début de l'histoire, le citoyen soviétique agit de manière patriotique, il s'en prend à la voiture de l'«ennemi capitaliste ». Puis renversement de situation : l'acte « antiaméricain » bascule dans un discours philoaméricain, le tout dans un contexte proche de l'absurde. Il est intéressant de noter que, dans la vie réelle, le Soviétique agit très exactement à l'opposé : en public, il adopte un discours antiaméricain, et en privé, il se comporte autrement (en recherchant des objets de consommation, par exemple). Si le ressort principal est l'absence de liberté en URSS, passe également l'idée que le citoyen soviétique ne peut sentir « l'air de la liberté » qu'en usant de stratégies paradoxales. En extrapolant, il est aisé de percevoir ici la trace de ce sentiment complexe d'amour et de haine qu'une partie des Soviétiques éprouve à l'égard des États-Unis : l'antiaméricanisme des médias peut donner envie de passer à l'acte contre le « principal ennemi de classe », mais ce rejet violent n'est que le revers d'un désir encore plus âpre de possession. Cette lutte, qui se révèle finalement un combat contre soi-même, passe par la volonté de rivaliser avec la première puissance industrielle du monde.

\section{Des rivalités}

Dans cette deuxième catégorie, il n'est plus seulement question de simple mise en parallèle entre deux mondes : l'accent est placé sur la compétition entre les deux puissances, le retard et les difficultés économiques soviétiques. Il semble évident que l'âge d'or de ces histoires drôles remonte à l'ère Hruščev, lorsque le Premier secrétaire, objet de risées pour son langage souvent fleuri, parlait de « rattraper et dépasser l'Amérique » (slogan emprunté à Stalin, mais qui date en réalité de Lenin). Plus que la rivalité économique, c'est la course spatiale, la plus spectaculaire, qui inspire les histoires les plus marquantes, dont l'une peut être datée de 1969-1970 :

Брежнев вызвал группу космонавтов.

- Товарищи! Американцы высадились на Луне. Мы тут подумали и решили, что вы полетите на Солнце!

- Так сгорим ведь, Леонид Ильич!

- Не бойтесь, товарищи, партия подумала обо всем. Вы полетите ночью. ${ }^{14}$

13. Un Soviétique se précipite vers la voiture de l'ambassadeur américain et crève un pneu.

« J'ai eu envie de respirer l'air de la liberté ! », explique-t-il.

14. Brežnev convoque un groupe de cosmonautes.

- Camarades ! Les Américains se sont posés sur la Lune. On a réfléchi : on a décidé que vous iriez sur le Soleil!

- Mais on va brûler, Leonid Il'ič !

— N'ayez crainte, camarades, le Parti a pensé à tout : vous partirez de nuit. 
Dans la tradition héritée de Hruščev, les Américains donnent l'occasion de brocarder la sénilité de Brežnev - personnage qui a dépassé de loin son prédécesseur comme sujet des anecdotes. Il est donc moins question ici de rivalité soviétoaméricaine dans l'espace que de la stupidité du pouvoir. Mais le rire masque une inquiétude : que va-t-il se passer si on confie de telles technologies, voire la direction du pays, à des hommes aussi incompétents ? On retrouve ici le très fort mépris des intellectuels soviétiques pour les dirigeants du pays.

La rivalité peut également concerner le sens des mots, seule la situation décrite dans l'anecdote exprime cet aspect conflictuel. Deux histoires ont pour ressort la question essentielle de la liberté et de sa signification pour les deux pays. La critique du détournement sémantique des mots par la propagande se retrouve dans ce dialogue connu. Bien que l'origine en remonte à l'administration Eisenhower, la mention de Reagan prouve que l'anecdote circule bel et bien pendant notre période :

- У нас свобода, - говорит американец. - Я могу выйти на улицу и кричать « Долой Рейгана !»

- Подумаешь! - говорит русский. - Я тоже могу выйти на улицу и кричать « Долой Рейгана! »15

L'histoire parodie les débats sur les libertés démocratiques. L'essentiel consiste donc ici en la dénonciation de la propagande soviétique, plus qu'en l'apologie de la liberté d'expression américaine qui demeure toutefois un élément important, dans la mesure où la diffusion même de l'histoire démontre qu'elle est évidente. Cette histoire appartient à un groupe d'anecdotes plus large, celui de la dénonciation de la propagande orale, dans le cadre de «conférences» (lekcii et politinformacii), « leçons politiques » auxquelles devaient obligatoirement assister les employés de toute entreprise soviétique :

На политзанятиях лектор говорит рабочим :

- Американские агрессоры угрожают нам своим новым оружием нейтронной бомбой. После её взрыва население гибнет, но всё остаётся целым. Рабочий (с места) :

- А какая бомба взорвалась у нас, что мы все остались целы а в магазинах ничего нет ? ${ }^{16}$

L’objet raillé en premier est la crise économique soviétique. De graves carences d'approvisionnement, apparues à la fin des années 1970, deviennent criantes dans

15. Nous sommes libres, dit l'Américain. Je peux sortir dans la rue et crier «Reagan, démission!»

- Et alors ?, répond le Russe, moi aussi, je peux sortir dans la rue et crier «Reagan, démission!»

16. Pendant le cours politique, le conférencier dit aux ouvriers : «Les agresseurs américains nous menacent de leur nouvelle arme - la bombe à neutrons. Après son explosion, toute la population meurt, mais les objets restent intacts ».

Un ouvrier : «Et quelle bombe a donc explosé chez nous que nous sommes restés en vie et que les magasins sont vides?» 
la première partie des années 1980. La rivalité militaire est également cruciale : la « bombe à neutrons » («bombe à radiation renforcée ») est un épouvantail récurrent de la propagande soviétique sous la présidence Carter (elle fait cependant partie de l'arsenal américain dès 1974), puis surtout avec Reagan, qui relance sa production. Pendant toute cette période, les médias soviétiques ne cessent de mener une campagne, la majorité de la population en est littéralement saturée. Celle-ci n'a peut-être pas bien compris de quoi il s'agissait précisément, mais à notre sens il ne fait pas de doute que l'expression «bombe à neutrons » fait partie du vocabulaire courant. La propagande officielle est ici détournée : on parle de bombe à neutrons, alors que les Soviétiques ont leur propre bombe et que les dirigeants s'avèrent incapables d'assurer l'approvisionnement des magasins.

D'autres histoires de ce groupe soulignent la dépendance économique de l'URSS des États-Unis (notamment l'achat de céréales ${ }^{17}$ ). Enfin, certaines parodient les questions soi-disant posées à la radio arménienne (armjanskoe radio) ${ }^{18}$ :

- Будет ли третья мировая война ?

- Не будет. Но будет такая борьба за мир - камня на камне не останется. ${ }^{19}$

L'anecdote s'inscrit probablement dans le contexte des nombreuses campagnes soviétiques «pour le désarmement » qui se multiplient dans la seconde partie des années 1970, et atteignent un nouveau point culminant au début des années 1980 . L'hypocrisie de ces campagnes est patente, puisque l'URSS ne cesse de parler de paix tout en renforçant son potentiel militaire. Le contraste est criant entre l'omniprésence du mot «paix » dans les discours officiels, culminant lors la crise des euromissiles en 1979-198320, et la course aux armements, bien réelle.

Cette histoire marque cependant un tournant : ce n'est plus seulement l'URSS, sa propagande mensongère ou ses dirigeants séniles qui sont seuls mis au pilori. Les États-Unis comme l'URSS sont coupables de pratiquer un discours que contredisent leurs actions. Cette rivalité cachée ne peut mener qu'à la destruction.

17. Plusieurs accords sur l'achat de blé américain sont signés à partir des années 1970, mais le record tombe en octobre 1984, avec Reagan proposant de relever les ventes de 12 à 22 millions de tonnes.

18. Voir Šmeleva et Šmelev, Russkij anekdot..., p. 98-108. Les anecdotes sur la « radio arménienne » apparaissent dans les années 1960, à l'apogée de la popularité de la radio comme médium. Une partie des émissions était composée de questions censées être posées par les auditeurs, en réalité souvent inventées de toutes pièces pour légitimer des mises au point idéologiques. Le qualificatif « arménien » viendrait des devinettes arméniennes aux réponses souvent surprenantes, populaires dans la Russie du début du $\mathrm{XX}^{\mathrm{e}}$ siècle. Un rare recueil d'anecdotes soviétiques consacré uniquement à Radio Arménie a été publié en 1970 par Nikolaj Olin (de son vrai nom Men'čukov, 1909-1977), Govorit «Radio Erevan » : Izbrannye voprosy i otvety [Ici Radio Erevan : Recueil des meilleures questions et réponses].

19. - Y aura-t-il une troisième guerre mondiale ?

- Non. Mais il y aura une telle lutte pour la paix qu'il ne restera pas une pierre.

20. Rappelons que la décision de l'OTAN de déployer les Pershing II en Europe occidentale date de décembre 1979; en octobre 1983 eut lieu une immense «marche pour la paix » à Moscou et dans d'autres villes soviétiques. 
L'ironie mordante de ces histoires drôles se retrouve dans d'autres anecdotes qui dépeignent une Amérique irrémédiablement étrangère à la réalité russe, et cette différence est visible à l'œil nu :

Подготовили в США агентов-разведчиков, обучили всему, что надо, даже русским провинциальным диалектам и забросили в СССР. Один из них вышел из леса в деревню и попросил старушку дать напиться. Старуха вынесла молока и говорит :

- Пей, сынок. А что у вас в Америке коровы перестали доить ?

- С чего ты взяла, бабушка, что я из Америки ?

- Что я слепая ? Я ж вижу, что ты негр. ${ }^{21}$

L'espionnite en URSS, cultivée dans la littérature et au cinéma surtout depuis les années 1950, retrouve un nouveau souffle en 1982-1985: dans ce contexte, l'histoire montre que la puissance technologique des États-Unis n'est rien face au «bon sens » de simples Russes (mais non de l'État soviétique). On retrouve aussi ici le cliché qui identifie souvent l'Américain au « Noir ». C'est l'inversion d'une propagande officielle pour laquelle les Afro-Américains, victimes du système américain, donc amis des Russes, sont incapables d'espionner au profit des capitalistes. Globalement, ces nouvelles tendances des anecdotes se retrouvent dans le groupe d'histoires où Soviétiques et Américains se rencontrent au sommet.

\section{Rencontres au sommet}

Ce dernier groupe est prépondérant. C'est là aussi que les histoires sont le plus réutilisables en fonction du contexte, s'adaptant à chaque nouveau protagoniste. Nous avons tenté d'approcher ce phénomène en relevant le nombre total de substitutions des anecdotes prêtées à un autre personnage ; nous remontons ainsi au début des années 1970 :

\begin{tabular}{|l|c|c|c|c|}
\cline { 2 - 5 } \multicolumn{1}{c|}{} & Kissinger & Nixon & Carter & Reagan \\
\hline Total des anecdotes & 2 & 6 & 11 & 13 \\
\hline $\begin{array}{l}\text { Nombre } \\
\text { de substitutions }\end{array}$ & Carter $: 1$ & $\begin{array}{c}\text { Kennedy }: 1 \\
\text { Carter }: 2\end{array}$ & $\begin{array}{c}\text { Kennedy }: 1 / \\
\text { Kissinger }: 1 / \\
\text { Nixon }: 2 / \text { Reagan }: 2\end{array}$ & Carter $: 2$ \\
\hline $\begin{array}{l}\text { Total des anecdotes } \\
\text { «pures » } \\
\text { (voir ci-dessous) }\end{array}$ & 1 & 3 & 5 & 11 \\
\hline
\end{tabular}

21. Les États-Unis ont préparé et entraîné des agents d'infiltration pour l'URSS : on leur a tout appris, même les dialectes provinciaux, et on les a parachutés sur le terrain. L'un d'entre eux sort de la forêt dans un village et demande à boire à une vieille. La vieille lui sort du lait et dit :

- Bois, mon fils. Et quoi, chez vous en Amérique les vaches n'ont plus de lait?

- D'où tiens-tu que je viens d'Amérique?

- Je ne suis pas aveugle, je vois bien que tu es Noir. 
La présence d'Henry Kissinger dans les anecdotes qui circulent au cours de la « guerre fraîche» peut surprendre. Au cours d'une carrière exceptionnellement longue autant qu'active sous deux présidences, ce diplomate vint à maintes reprises à Moscou, avant d'être désavoué par le Congrès en 1976. La presse soviétique des années 1960-1970 reprend inlassablement le mythe de sa toute-puissance en matière de politique étrangère. Il n'est donc guère étonnant que l'une des anecdotes ait pour cadre une rencontre avec Brežnev, dans laquelle est dénoncée la domination soviétique en Europe de l'Est ${ }^{22}$, et qu'une autre, plus célèbre, évoque le principe de la « diplomatie de la navette » (čelnočnaja diplomatija), appliquée au cas d'un paysan russe 23 :

Валентин Зорин беседует с Генри Киссинджером.

- Скажите, господин Киссинджер, вы считаетесь изобретателем «челночной дипломатии ». Поясните, что это такое, на примере?

- О, это очень просто, - отвечает Киссинджер.- допустим, прихожу я к Рокфеллеру и говор «Послушайте, Рокфеллер, вы хотите иметь своим зятем крутого сибирского мужика? »

- Фу ! говорит Рокфеллер. Вы что?

- А если он при этом будет клиентом швейцарского банка?

- О, это другое дело!

Дальше совсем просто. Прихожу я в банк, спрашиваю: «Вы хотите иметь своим клиентом крутого сибирского мужика?»

- Фу-у, говорят в банке.

- А если он при этом будет зятем Рокфеллера?

- О-о, другое дело! Дальше - просто пустяки. Приежаю к крутому сибирскому мужику, спрашива «Хочешь жениться на обыкновенной американке?»

- Фу-у!- говорит тот.

- А стать при этом зятем Рокфеллера?

- О-о! Другое дело! Теперь - сущая ерунда. Прихожу я к дочери Рокфеллера, спрашиваю:

- Ты хочешь иметь мужем клиента швейцарского банка?

- Фу-у, - говорит она.

- А если это будет крутой сибирский мужик?

- O-O-O-o! ${ }^{24}$

22. On peut donc la dater de la rencontre qui a suivi l'invasion de la Tchécoslovaquie en août 1968 .

23. Kissinger commence à être connu en URSS à partir de la fin des années 1960, par l'entremise du journaliste de télévision Valentin Zorin donc bien avant de devenir secrétaire d'État en 1973, voir les mémoires de Zorin, Neizvestnoe ob izvestnom, M. : Vagrius, 2000, p. 207. Le personnage et la popularité de Kissinger s'expliquent peut-être par ses origines juives (les anecdotes sur les juifs soviétiques sont un genre très riche). Comme l'a remarqué Amandine Regamey, la consonance même de son nom, voire d'autres protagonistes américains - les nommer, c'est déjà faire de l'humour - pourrait avoir contribué au succès de ces anecdotes.

24. Valentin Zorin fait une interview de Kissinger: « Dites-moi, Monsieur Kissinger, vous êtes considéré comme l'inventeur de la 'diplomatie de la navette'. Pouvez-vous expliquer cela par un exemple? »

- Mais c'est très simple, répond Kissinger. Supposons que je vienne chez Rockefeller et lui dise : «Écoutez, Rockefeller, voulez-vous être le beau-père d'un vigoureux paysan de Sibérie ? » 
Cette histoire, centrée sur l'énergie fantastique de l'Américain, non sur les défauts soviétiques, constitue une exception. Ailleurs, ce sont les chefs de l'exécutif américain qui sont surtout présents dans les anecdotes.

Des quatre présidents de la période, trois ont marqué les esprits des Soviétiques. Si l'on se fie aux chiffres absolus, Reagan semble avoir été le plus populaire. Ceci est confirmé par le nombre de ce que nous appelons des « anecdotes pures », celles qui ont appartenu à une époque donnée sans ensuite être reprises avec d'autres protagonistes. Ces anecdotes « pures » sont obtenues en soustrayant les « substitutions » du total. Ainsi, pour Reagan, une histoire qui fait explicitement référence à la guerre des Malouines :

Англичане захватили Фолклендские острова, а что делать с ними не знают. Спросили Рейгана:

- Не знаю, - ответил Рейган, - спросите у Брежнева, это он специалист по малым землям. ${ }^{25}$

Nous constatons ainsi que les anecdotes « pures » utilisant Reagan sont deux fois plus nombreuses que les « Carter». Naturellement, ces chiffres ne peuvent être considérés comme intangibles - il est certain que les substitutions furent plus nombreuses ${ }^{26}$. Il reste que cette supériorité numérique de «Reagan» sur «Carter» et, dans une moindre mesure, de «Carter» sur «Nixon» reflète, à notre avis, le contexte historique.

Tout d'abord, les anecdotes qui utilisent Nixon, bien qu'elles concernent au départ une période antérieure, n'en continuent pas moins à circuler au cours de notre décennie ;

- Beurk ! Vous voulez plaisanter?

- Et s'il était en même temps détenteur d'un compte dans une banque suisse ?

- Ah ça, c'est autre chose !

Ensuite, c'est très simple. J'arrive dans la banque suisse et je demande :

- Vous voulez avoir pour client un vigoureux paysan de Sibérie?

- Beurk!

— Et s'il était en même temps le gendre de Rockefeller ?

- Ah ça, c'est autre chose !

Ensuite c'est encore plus simple. Je vais voir le paysan de Sibérie :

- Tu veux épouser une simple Américaine?

- Beurk!

- Et devenir en même temps le gendre de Rockefeller?

- Ah ça, c'est autre chose !

Je vais voir la fille de Rockefeller :

- Tu veux avoir pour époux le client d'une banque suisse?

- Beurk!

- Et si c'était un vigoureux paysan de Sibérie ?

- Ah ça, c'est autre chose !

25. Les Anglais ont occupé les Malouines mais ne savent pas quoi en faire. Ils posent la question à Reagan :

- Je ne sais pas, répond Reagan demandez à Brežnev, c'est lui le spécialiste des Petites Terres.

26. Outre les recueils d'anecdotes susmentionnés, les mémoires des contemporains représentent une autre source essentielle : ainsi le journal inédit d'un haut fonctionnaire du Comité central du Parti communiste, Anatolij Černjaev, dans une note de mars 1980, signale qu'à Moscou circule « une anecdote sur le shah d'Iran » («Comment garantir à l'URSS des arrivées de pain ? - Échanger Sakharov contre le Shah, le Shah contre les diplomates américains, les diplomates contre des céréales »). 
toutes relèvent de la thématique de la confrontation idéologique des deux mondes. Comme nous l'avons vu, Nixon sert de révélateur des tares du système soviétique :

Посетивший СССР Никсон спросил у Брежнева, почему советские рабочие не бастуют. Вместо ответа Брежнев повез Никсона на завод и там обратился к рабочим:

- С завтрашнего дня вам будет уменьшена зарплата! (аплодисменты) Будет увеличен рабочий день! (аплодисменты) Каждого десятого будут вешать! (аплодисменты, вопрос: « веревку свою приносить или профком обеспечит? » $)^{27}$

Toutes les anecdotes dont il est l'un des protagonistes reposent sur un contexte avéré de rencontres régulières entre les chefs d'État des deux pays : en mai 1972 Nixon se rend à Moscou, en juin 1973 Brežnev est reçu à Washington, en juillet 1974 Nixon est de nouveau en URSS : voilà qui marque l'imaginaire collectif. L'ancrage solide dans le réel des anecdotes nixoniennes s'explique donc aisément par la large couverture des rencontres par les médias soviétiques.

Après un Gerald Ford absent du corpus - un trop court passage à la présidence n'a pu marquer les esprits soviétiques - , la présidence de Carter amorce un tournant, pas encore une rupture. Au vu des substitutions (au nombre de cinq), les anecdotes utilisant Carter sont parmi les plus recyclées. Viennent ensuite, loin derrière, Kennedy, Nixon et Reagan (deux). Carter reçoit l'héritage de cinq anecdotes (une de Kennedy et quatre de l'ère Nixon). Le corpus d'anecdotes Carter n'est donc pas entièrement original : comme pour Nixon, il repose en majorité sur le principe de la rencontre. La différence principale est la localisation : Carter a certes rencontré Brežnev à Vienne en juin 1979 pour la signature du traité SALT II, mais l'atmosphère n'est plus la même - Carter a entrepris depuis son accession à la présidence une farouche attaque sur le front du non-respect des droits de l'homme en URSS. On ne trouve par conséquent qu'une seule histoire qui mentionne spécifiquement Vienne, mais son anachronisme la date bien de $1980^{28}$ :

Картер спрашивает Брежнева, во время встречи в Вене:

- Почему ваши войска вошли в Афганистан?

- Потому что начинается на 'A' !

- Разве это достаточно для вторжения в суверенную страну ?

- А мы их - по алфавиту !29

27. Nixon en visite en URSS demande à Brežnev pourquoi les ouvriers soviétiques ne font jamais grève. Au lieu de lui répondre, Brežnev l'emmène dans une usine et s'adresse aux ouvriers :

- À partir de demain, votre salaire sera baissé ! (Applaudissements). La journée de travail sera prolongée ! (Applaudissements) Un ouvrier sur dix sera pendu ! (Applaudissements et question : «Faut-il apporter la corde soi-même, ou bien le comité d'entreprise la fournira-t-il ? »)

28. L'invasion de l'Afghanistan eut lieu fin décembre 1979, l'annonce dans les médias soviétiques ( « les troupes soviétiques sont venues en aide au régime ») en janvier 1980.

29. Carter demande à Brežnev, pendant la rencontre de Vienne :

- Pourquoi vos troupes sont-elles entrées en Afghanistan?

- Parce qu'il commence par 'A'!

- Et cela suffit-il pour envahir un pays ?

- On a choisi l'ordre alphabétique ! 
D'autres anecdotes de la période Carter ont pour point de départ des rencontres imaginaires. Le ton se fait beaucoup plus libre, le « respect» envers les grands de ce monde disparaît, on les place dans des situations parfois grotesques. Le dialogue emprunte désormais beaucoup plus au langage parlé, et la trame de l'anecdote n'est plus réellement politique. Certaines histoires témoignent à notre sens clairement d'un accaparement de l'anecdote politique par un public de jeunes de plus en plus indifférents à la politique et aux enjeux de la rivalité Est-Ouest ou, au contraire, d'un patriotisme grossier nouveau : ainsi dans une histoire où l'occupation de la Pologne est justifiée, Brežnev est fier d'inspirer la peur au monde entier.

En général, le souci de vraisemblance historique n'est plus respecté. L'anecdote suivante, qui daterait de l'ère Kennedy, retrouve une nouvelle jeunesse dans le contexte des Jeux olympiques de Moscou :

Джимми Картер и Л. И. Брежнев соревновались в беге на атлетической дорожке. Картер, естественно, добежал первым. Через полчаса до финиша с большим трудом доковылял Брежнев. На следующее утро сообщение TACC: « По результатам забега президент США Джимми Картер занял предпоследнее место. Генеральный секретарь ЦК КПСС Леонид Ильич Брежнев занял почетное второе место ». ${ }^{30}$

Au-delà d'une critique de la propagande soviétique, il y a là référence à un contexte de rivalité précis, celui du boycott des Jeux olympiques de Moscou. Sans ce référent, l'histoire perd tout son sel (le prétexte est aussi important que le texte luimême).

Quelles sont alors les innovations de cette période ? Sous la présidence Carter, le seul élément nouveau vraiment ancré dans le réel est l'apparition d'un « danger commun » aux deux puissances, la Chine. Deux anecdotes en effet évoquent l'invasion de l'URSS par la Chine et les dangers du désarmement face à cet ennemi potentiel. En voici une :

Брежнев с Картером сидят и едят кашу из одной миски. Брежнев съедает ложку каши, облизывает и бьет этой ложкой по лбу Картера со словами: «Довооружался!». Картер съедает ложку каши, облизывает, и со смаком, бьет ложкой по лбу Брежнева со словами: «Доразоружался!». В этот момент открывается дверь, заходит китаец с автоматом, и говорит : «Обед закончен! Всем по камерам!» ${ }^{31}$

30. Jimmy Carter et Leonid Brežnev se lancent dans l'épreuve du 100 mètres. Carter, naturellement, arrive premier. Une demi-heure plus tard, avec beaucoup d'efforts, arrive finalement Brežnev. Le lendemain, TASS informe : «D'après les résultats de la course, le président des États-Unis Jimmy Carter a remporté l'avant-dernière place. Le Secrétaire général du CC du PCUS Leonid Il’ič Brežnev occupe une honorable seconde place ».

31. Brežnev et Carter sont assis et mangent de la kaša dans un même bol. Brežnev avale une cuillerée, lèche la cuillère et en frappe Carter au front en disant : «Ça t'apprendra à t'armer ! » Carter fait de même, frappe Brežnev au front en disant : « Ça t'apprendra à désarmer ! » À ce moment la porte s'ouvre sur un Chinois armé qui dit: «Déjeuner terminé ! Chacun dans sa cellule !» 
Rappelons que le conflit avec la Chine remonte en URSS au XXe Congrès (1956) et à la déstalinisation, mais franchit un seuil purement idéologique en mars 1969 avec les incidents meurtriers sur l'Oussouri. La mort de Mao en 1976 n'entraîne pas de réchauffement dans les relations : au cours des années 1970 les États-Unis se servent de la Chine comme d'un « atout » pour faire pression sur l'URSS. Début 1979, Deng Xiaoping se rend aux États-Unis d'où il dénonce « l'hégémonie soviétique ». Avant Reagan, la Chine est donc vilipendée dans les médias soviétiques de manière beaucoup plus agressive (quoique plus rarement) que les États-Unis. Il ne fait pas de doute qu'elle représente dans les anecdotes un danger bien plus imminent que les ÉtatsUnis par rapport auxquels une entente ancienne (dans les domaines économique, militaire et culturel) tend à diminuer les craintes d'une troisième guerre mondiale. En général, à partir de l'ère Carter, l'inquiétude face à l'avenir en même temps qu'une certaine « dépolitisation» sont les deux traits majeurs des anecdotes ${ }^{32}$.

Ces éléments, combinés à d'autres déjà évoqués, inspirent les anecdotes reaganiennes. Au cours de son premier mandat, bien qu'aucune rencontre au sommet n'eût lieu, l'éventualité en fut évoquée ouvertement ${ }^{33}$. Deux histoires mentionnent la rencontre des deux hommes (trahissant de fait la jeunesse de leurs auteurs). Une autre parle de la crainte qu'éprouve Reagan de rencontrer Brežnev. Mais la majorité des histoires «s'accrochent » à la réalité par le biais de détails : communication téléphonique, absence d'un des deux protagonistes... Parfois un élément onirique ou fantastique est introduit dans un contexte d'après-troisième-guerre mondiale :

На земном шаре остались два человека - Брежнев и Рейган. И вот Рейган берет огромный камень, подкрадывается тихонечко сзади к Брежневу. В этом время кто-то его ломом бац по спине. Вопрос: « Кто это был? » Ответ: « Вечно живой Ильич». ${ }^{34}$

Le détournement d'une préoccupation angoissante, le danger d'une confrontation nucléaire, est ainsi discrètement suggéré dans les anecdotes de ce dernier groupe. Constatons que la période d'extrême tension entre les deux pays, qui atteint son

32. Pour ce qui est de l'apolitisme croissant, une lettre anonyme d'un lecteur de quinze ans de la région de Sverdlovsk, envoyée à la Pionerskaja Pravda en 1979, donne le ton d'un état d'esprit largement partagé qui va aller s'amplifiant : «L'intérêt pour les plaisirs simples a supplanté les intérêts sérieux, nous n'avons plus besoin de la politique, nous ne nous intéressons pas aux stakhanovistes de l'industrie et au bilan du plan quinquennal - nous voulons danser, donneznous du rock! » RGASPI (Rossijskij gosudarstvennyj arhiv social’no-političeskoj istorii Archives d'histoire sociopolitique de la Fédération de Russie), f. 1m, inv. 95, d. 54a, f. 6. Voir d'autres exemples dans la conclusion ci-après.

33. D'après les mémoires de l'ambassadeur soviétique aux États-Unis Anatolij Dobrynin, Brežnev lance la proposition début mars 1981. A. Dobrynin Sugubo doveritel'no. Posol v Vašingtone pri šesti presidentah (1962-1986) [Hautement confidentiel. Ambassadeur à Washington pendant six mandats présidentiels], M. : Avtor, 1996,. Mais c'est en avril 1982 que Reagan reprend l'idée officiellement et Brežnev le soutient dans la Pravda (le 18 avril), évoquant l'éventualité d'une rencontre en octobre.

34. Deux hommes seulement ont survécu sur terre: Brežnev et Reagan. Reagan prend une grosse pierre, s'approche silencieusement de Brežnev par-derrière. À ce moment, quelqu'un le frappe dans le dos. «Qui était-ce ? ». Réponse : «Vladimir Il’ič, éternellement vivant ! » 
apogée sous Andropov fin 1983-début 1984, trouve un écho dans l'utilisation d'un procédé fantastique : la rencontre dans un ailleurs postapocalyptique. La politisation des anecdotes ancrées dans un contexte historique précis n'est plus de mise, ou ne suscite plus le sourire ; sa gravité est liée à son imminence : on ne trouve pas d'anecdotes sur les sanctions américaines contre l'URSS dans le cadre des « événements » de Pologne, ou sur la destruction par les Soviétiques de l'avion sudcoréen en 1983 ; ni sur le déploiement des missiles américains et soviétiques en Europe occidentale. On n'exploite pas l'exécrable « plaisanterie » d'un Reagan qui pensait être hors antenne à la radio, le 11 août 1984 : «Nous allons bombarder la Russie dans cinq minutes ». Certes, en règle générale, la gravité d'une situation n'arrête pas les blagueurs, mais manifestement aucun de ces événements ne les a inspirés. Mais notre corpus peut-il être considéré comme représentatif ? Un désintérêt croissant de la population pour la chose politique, dans les années 1970-1980, serait-il la raison principale de cet état de fait? La question reste ouverte.

$$
* * *
$$

Quel bilan dresser de notre analyse ? L'image des États-Unis et des Américains que donne l'anecdote politique soviétique est difficile à cerner car ni les uns ni les autres n'en sont les protagonistes principaux. Ils servent à l'évidence d'abord de faire-valoir aux défauts soviétiques, notamment l'hypocrisie de la propagande. En cela, ils contribuent à façonner « l'anti-mythe soviétique » décrit par Seth Graham ${ }^{35}$. On peut même aller plus loin : poser qu'un certain « complexe d'infériorité » expliquerait l'émergence de ces histoires. Ce complexe se renforce dans les années 1970 quand une majorité de la société partage le désenchantement face à un illusoire «socialisme réel ${ }^{36}$. On ne rit pas que du système mais surtout de soi : ce rire ne veut cependant pas dire que l'on rejette le « socialisme réel » au profit d'un hypothétique capitalisme dont on ne sait pas grand-chose. C'est donc d'une quête identitaire que participent ces anecdotes : à la lancinante question « qui sommes-nous ? », la présence américaine apporte une réponse partielle : «nous ne sommes pas comme eux », ce qui dans les anecdotes plus tardives se colore d'un sentiment de fierté. Cette construction d'une identité négative, entre autoflagellation et fierté nationale, bien analysées dans les travaux du sociologue Lev Gudkov ${ }^{37}$, est l'élément essentiel que l'on peut retirer de l'analyse des anecdotes. Croisées avec d'autres sources, elles confirment l'ambivalence de l'attitude envers l'Amérique - admirée et haïe.

35. Voir sa thèse soutenue en 2003 à l'Université de Pittsburgh : « A Cultural Analysis of the Russo-Soviet Anekdot»,p. 6.

36. A. V. ŠubIN, Ot «Zastoja » k reformam: SSSR v 1917-1985 gg. [De la « stagnation » aux réformes : L'URSS de 1917 à 1985], M. : Rosspen, 2001.

37. Lev Gudkov, Negativnaja identičnost'. Stat'i 1997-2002 [L'identité négative. Articles, 1997-2002]. M. : NLO, 2004. Ce recueil d'articles contient deux textes essentiels : « Otnošenie k SŠA v Rossii i problema antiamerikanizma » [L'attitude envers les États-Unis en Russie et la question de l'antiaméricanisme], p. 496-551 et «Ideologema «vraga » » [Idéologème de l'ennemi], p. 552-649. 
Le deuxième élément que nous pouvons distinguer est d'ordre diachronique. Avec le temps, le contexte aidant, philoaméricanisme et rejet du système soviétique comme mensonger cèdent la place à un certain relativisme. Les États-Unis partagent avec l'URSS la responsabilité de la montée des tensions dans le monde, tensions susceptibles de déboucher sur un conflit nucléaire. Les anecdotes nous confirment indirectement que la peur fut bien réelle. D'autres sources vont dans le même sens, par exemple les nombreuses lettres et pétitions adressées au Comité central du VLKSM pendant la préparation de la marche pour la Paix ${ }^{38}$.

Enfin, l'anecdote semble corroborer l'idée d'une opinion publique différenciée en fonction des générations. Si les adultes racontent des anecdotes entre eux, le plus souvent à la cuisine, lieu privilégié légendaire de l'élaboration d'une protoopinion publique ${ }^{39}$, plusieurs témoignages font état de jeunes qui à l'école multiplient sans complexe les plus scabreuses anecdotes mettant en scène l'idéologie communiste ou les dirigeants du pays ${ }^{40}$. Pour eux, il ne s'agit pas seulement d'évacuer l'excessive pression de l'idéologie ${ }^{41}$, mais surtout d'une provocation qui ne vise pas à diffuser un quelconque message politique. Ce qu'une partie de la jeune génération rejette avant tout - le courrier des jeunes lecteurs susmentionné nous l'apprend c'est un optimisme de commande largement discrédité.

En tout cas, ces anecdotes confirment une idée : l'opinion sur les États-Unis formée indépendamment de la propagande officielle est inconcevable en URSS. L'information officielle sert toujours de référentiel : concevoir l'Amérique sans elle est, pour une immense majorité de ceux qui n'y sont jamais allés, impossible. Il en résulte que le philoaméricanisme apparu dans les années 1960 est aussi (et surtout, peut-être) un philoaméricanisme d'opposition. Né du rejet de la propagande, il n'exclut nullement des manifestations d'antiaméricanisme chez les mêmes personnes. Ceci expliquerait le succès des sentiments antiaméricains dans la Russie actuelle, avec un Putin si populaire. Au final, la blague politique, sans être une source de premier ordre, n'apporte pas moins des éléments importants à une enquête sur la société soviétique.

Centre d'études des mondes russe, caucasien et centre-européen EHESS

kozovoi@gmail.com.

38. RGASPI (f.m1, inv.95, d.304).

39. Dmitriev, Sociologija političeskogo jumora [Sociologie de l'humour politique], M. : Rosspen, 1998,p. 41.

40. Zajceva, élève de 9e classe, signale dans un courrier adressé à Komsomol'skaja Pravda en 1983, année de l'apogée de la « guerre fraîche », que dans son école « on peut même entendre ce genre d'anecdotes [politiques] dans la bouche des militants [du Komsomol] [...] Et si on essaye de les arrêter, on entend en réponse - « encore toi avec ta morale ». Tout et tout le temps est soumis à une stupide dérision », RGASPI : 1m, inv.95, d.267, f.78. De même, l'alcool aidant, les jeunes ne se gênent pas pour les raconter ostensiblement dans la rue, voir RGASPI, f.1m, inv.95, d.267, f.70-71. À noter que tous ces témoignages proviennent de la province.

41. Dmitriev, Sociologija..., p. 57. 